Research Article

Special Issue: Combinatorial Matrices

Open Access

Mohammad Reza Oboudi

\title{
Seidel energy of complete multipartite graphs
}

https://doi.org/10.1515/spma-2020-0131

Received October 30, 2020; accepted March 18, 2021

Abstract: The Seidel energy of a simple graph $G$ is the sum of the absolute values of the eigenvalues of the Seidel matrix of $G$. In this paper we study the Seidel eigenvalues of complete multipartite graphs and find the exact value of the Seidel energy of the complete multipartite graphs.

Keywords: Seidel matrix of graphs; Seidel energy of graphs; Complete multipartite graphs

MSC: 05C31, 05C50, 15A18, 15A42

\section{Introduction}

Throughout this paper we will consider only simple graphs ( finite and undirected, without loops and multiple edges). Let $G=(V(G), E(G))$ be a simple graph. The order of $G$ denotes the number of vertices of $G$. The complement of $G$, denoted by $G$, is the simple graph with vertex set $V(G)$ such that two distinct vertices of $G$ are adjacent if and only if they are not adjacent in $G$. The edgeless graph (empty graph) and the complete graph of order $n$, are denoted by $K_{n}$ and $K_{n}$, respectively. Let $t$ and $n_{1}, \ldots, n_{t}$ be some positive integers. By $K_{n_{1}, \ldots, n_{t}}$ we mean the complete multipartite graph with parts size $n_{1}, \ldots, n_{t}$. In particular the complete bipartite graph with part sizes $m$ and $n$ is denoted by $K_{m, n}$. The identity matrix of size $n$ is denoted by $I_{n}$. By $J_{n}$ we mean the $n \times n$ matrix whose all of entries are 1 . We use $I$ and $J$ instead of $I_{n}$ and $J_{n}$, respectively, if the size of them are clear from the text. For every Hermitian matrix of size $n$, say $A$, we sort its eigenvalues as $\lambda_{1}(A) \geq \lambda_{2}(A) \geq \cdots \geq \lambda_{n}(A)$. We recall that a Hermitian matrix (or self-adjoint matrix) is a complex square matrix that is equal to its own conjugate transpose. The eigenvalues of Hermitian matrices are real. For every real square matrix $B$, the trace of $B$, denoted by $\operatorname{tr}(B)$, is defined to be the sum of the entries on the main diagonal of $B$. The rank of $B$ is denoted by $\operatorname{rank}(B)$.

Let $G$ be a simple graph with vertex set $\left\{v_{1}, \ldots, v_{n}\right\}$. The adjacency matrix of $G$, denoted by $A(G)$, is the $n \times n$ matrix such that the $(i, j)$-entry is 1 if $v_{i}$ and $v_{j}$ are adjacent, and otherwise is 0 . The Seidel matrix of $G$ that is denoted by $S(G)=\left[s_{i j}\right]$ is a $n \times n$ matrix in which

$$
s_{i j}:= \begin{cases}0, & \text { if } i=j ; \\ -1, & \text { if } i \neq j, \text { and } v_{i} \text { and } v_{j} \text { are adjacent; } \\ 1, & \text { if } i \neq j, \text { and } v_{i} \text { and } v_{j} \text { are not adjacent. }\end{cases}
$$

We note that $S(G)=A(\bar{G})-A(G)$. In addition, since $A(\bar{G})=J_{n}-I_{n}-A(G)$, we obtain that $S(G)=J_{n}-I_{n}-2 A(G)$. The adjacency matrix and Seidel matrix are real and symmetric, so all of their eigenvalues are real. The Seidel characteristic polynomial of $G$, denoted by $\Psi(G, \theta)$, is the determinant of the matrix $\theta I_{n}-S(G), \operatorname{det}\left(\theta I_{n}-S(G)\right.$. By the eigenvalues of $G$ we mean those of its adjacency matrix. We denote the eigenvalues of $G$ by $\lambda_{1}(G) \geq$ $\cdots \geq \lambda_{n}(G)$. Similarly, by the Seidel eigenvalues of $G$ we mean those of its Seidel matrix. We denote the Seidel eigenvalues of $G$ by $\theta_{1}(G) \geq \cdots \geq \theta_{n}(G)$. We note that the Seidel eigenvalues of $G$ are the roots of the Seidel characteristic polynomial of $G$. The energy of a graph $G$, denoted by $\mathcal{E}(G)$, is the sum of the absolute values

Mohammad Reza Oboudi: Department of Mathematics, College of Sciences, Shiraz University, Shiraz, 71457-44776, Iran, E-mail: mr_oboudi@yahoo.com,mr_oboudi@shirazu.ac.ir 
of the eigenvalues of $G$, see [3] and [4]. In other words

$$
\mathcal{E}(G)=\left|\lambda_{1}(G)\right|+\cdots+\left|\lambda_{n}(G)\right| .
$$

Similarly, Haemers [5] defined the Seidel energy of $G$, that is denoted by $\varepsilon(S(G))$, as the sum of the absolute values of the eigenvalues of the Seidel matrix of $G$. In other words,

$$
\mathcal{E}(S(G))=\left|\theta_{1}(G)\right|+\cdots+\left|\theta_{n}(G)\right| .
$$

See [5],[8] and [9] for more details on Seidel energy of graphs. There are many other matrices associated to graphs such as Laplacian matrix and signless Laplacian matrix of graphs. One of the attractive graphs that the researchers study the properties of its associated matrices is the complete multipartite graphs, for instance see [1],[6],[7],[10],[11],[12] and [13].

In this paper we investigate the Seidel energy of the complete multipartite graphs. We find a formula for computing the Seidel energy of the complete multipartite graphs. More precisely, we show that for every positive integers $t \geq 2$ and $n_{1}, \ldots, n_{t}$,

$$
\mathcal{E}\left(S\left(K_{n_{1}, \ldots, n_{t}}\right)\right)=2 n-2 t-2 \theta_{n}\left(K_{n_{1}, \ldots, n_{t}}\right),
$$

where $n=n_{1}+\cdots+n_{t}$. As a consequence of the above formula, we obtain that among all complete multipartite graphs, the complete graphs have the minimum Seidel energy.

\section{A formula for the Seidel energy of complete multipartite graphs}

In this section we find a formula for computing the Seidel energy of complete multipartite graphs. First we recall the interlacing property and a result related to the eigenvalues of the summation of two Hermitian matrices.

Theorem 1. [Interlacing Theorem 2.5.1 of [2]] Let $A$ be a real symmetric matrix of size $n$ and $B$ be a principal submatrix of $A$ with size $m$. Suppose that $\lambda_{1}(A) \geq \cdots \geq \lambda_{n}(A)$ and $\lambda_{1}(B) \geq \cdots \geq \lambda_{m}(B)$ are the eigenvalues of $A$ and $B$, respectively. Then for every $i, 1 \leq i \leq m, \lambda_{i}(A) \geq \lambda_{i}(B) \geq \lambda_{n-m+i}(A)$. In particular $\lambda_{m}(B) \geq \lambda_{n}(A)$.

Theorem 2. [Courant-Weyl Inequalities,[2]] Let $A$ and $B$ be Hermitian matrices of size $n$, and let $1 \leq i, j \leq n$. Then the following hold.

(i) If $i+j-1 \leq n$, then $\lambda_{i+j-1}(A+B) \leq \lambda_{i}(A)+\lambda_{j}(B)$.

(ii) If $i+j-n \geq 1$, then $\lambda_{i}(A)+\lambda_{j}(B) \leq \lambda_{i+j-n}(A+B)$.

As a corollary of Courant-Weyl inequalities we obtain the following result.

Lemma 1. Let $A$ be a $n \times n$ symmetric matrix with real entries. Then

$$
\lambda_{1}(A+J) \geq \lambda_{1}(A) \geq \lambda_{2}(A+J) \geq \lambda_{2}(A) \geq \cdots \geq \lambda_{n-1}(A+J) \geq \lambda_{n-1}(A) \geq \lambda_{n}(A+J) \geq \lambda_{n}(A),
$$

where $J$ is the $n \times n$ matrix that all of entries are 1 .

Proof. There is nothing to prove for $n=1$. Now let $n \geq 2$. We note that $\lambda_{1}(J)=n$ and $\lambda_{2}(J)=\cdots=\lambda_{n}(J)=0$. Since $\lambda_{n}(J)=0$, by letting $j=n$ in the second part of Theorem 2, we obtain that for $i=1, \ldots, n$,

$$
\lambda_{i}(A+J) \geq \lambda_{i}(A)+\lambda_{n}(J)=\lambda_{i}(A) .
$$

On the other hand since $\lambda_{2}(J)=0$, by letting $j=2$ in the first part of Theorem 2 , we find that for $i=1, \ldots, n-1$,

$$
\lambda_{i+1}(A+J) \leq \lambda_{i}(A)+\lambda_{2}(J)=\lambda_{i}(A) .
$$

Now the result follows by combining the Equations (1) and (2). 
By the following result one can compute the Seidel characteristic polynomial of complete multipartite graphs.

Theorem 3. [13] Let $t \geq 2$ and $n_{1}, \ldots, n_{t}$ be some positive integers and $n=n_{1}+\cdots+n_{t}$. Then

$$
\Psi\left(K_{n_{1}, \ldots, n_{t}}, \theta\right)=(\theta+1)^{n-t}\left(\prod_{j=1}^{t}\left(\theta-2 n_{j}+1\right)+\sum_{j=1}^{t} n_{j} \prod_{i=1, i \neq j}^{t}\left(\theta-2 n_{i}+1\right)\right) .
$$

In sequel we will see that the smallest Seidel eigenvalue of complete multipartite graphs has essential role is our results. So first we obtain an upper bound for that.

Remark 1. We note that for every graph $G, S(G)=J-I-2 A(G)$. In particular, $S\left(K_{n}\right)=I-J=-A\left(K_{n}\right)$. Using the eigenvalues $J$, this shows that the Seidel eigenvalues of the complete graph $K_{n}$ ( $n$ is a positive integer) are 1 (with multiplicity $n-1$ ) and $1-n$ (with multiplicity 1 ). Thus $\mathcal{E}\left(S\left(K_{n}\right)\right)=2 n-2$. Using Theorem 3 , it is not hard to see that the Seidel eigenvalues of the complete bipartite graph $K_{p, q}$ ( $p$ and $q$ are positive integers) are $p+q-1$ (with multiplicity 1 ) and -1 (with multiplicity $p+q-1$ ). In particular $\mathcal{E}\left(S\left(K_{p, q}\right)\right)=2(p+q)-2$.

Since by Remark 1, the smallest Seidel eigenvalue of complete bipartite graphs is exactly determined (this parameter is equal to -1 ), it is remain to obtain an upper bound for the smallest Seidel eigenvalue of complete multipartite graphs that have at least three parts.

Theorem 4. Let $t \geq 3$ and $n_{1}, \ldots, n_{t}$ be some positive integers. Let $n=n_{1}+\cdots+n_{t}$ and $\theta_{1} \geq \cdots \geq \theta_{n}$ be the Seidel eigenvalues of $K_{n_{1}, \ldots, n_{t}}$. Then

$$
\theta_{n} \leq 1-t
$$

Moreover the equality holds if and only if $n_{1}=\cdots=n_{t}=1$.

Proof. First assume that $n_{1}=\cdots=n_{t}=1$. So $n=t$ and $K_{n_{1}, \ldots, n_{t}}$ is the complete graph $K_{t}$. Hence by Remark 1 , $\theta_{n}=\theta_{n}\left(K_{n_{1}, \ldots, n_{t}}\right)=\theta_{t}\left(K_{t}\right)=1-t$. Thus the equality holds for $n_{1}=\cdots=n_{t}=1$.

Now suppose that there exists $j \in\{1, \ldots, t\}$ such that $n_{j} \geq 2$. Without losing the generality assume that $n_{1} \geq 2$. Let $H_{t}=K_{2, \underbrace{1, \ldots, 1}_{t-1}}$. Since $H_{t}$ is an induced subgraph of $K_{n_{1}, \ldots, n_{t}}$, the Seidel matrix of $H_{t}$ is a principal submatrix of the Seidel matrix of $K_{n_{1}, \ldots, n_{t}}$. In other words $S\left(H_{t}\right)$ is a principal submatrix of $S\left(K_{n_{1}, \ldots, n_{t}}\right)$ (we note that for every graph $G, S(G)=J-I-2 A(G))$. Thus by Interlacing Theorem 1 we find that $\theta_{n}\left(K_{n_{1}, n_{2}, \ldots, n_{t}}\right) \leq$ $\theta_{t+1}\left(H_{t}\right)$. Hence to complete the proof it suffices to show that $\theta_{t+1}\left(H_{t}\right)<1-t$. Using Theorem 3 one can see that

$$
\Psi\left(H_{t}, \theta\right)=(\theta+1)(\theta-1)^{t-2}\left(\theta^{2}+(t-3) \theta+4-3 t\right)
$$

This shows that the Seidel eigenvalues of $H_{t}$ are 1 (with multiplicity $t-2$ ), -1 , and $\frac{3-t \pm \sqrt{t^{2}+6 t-7}}{2}$. Since $t \geq 3$, it is easy to see that $\theta_{t+1}\left(H_{t}\right)=\frac{3-t-\sqrt{t^{2}+6 t-7}}{2}<1-t$. This completes the proof.

Now we prove the main result of the paper.

Theorem 5. Let $t \geq 2$ and $n_{1}, \ldots, n_{t}$ be some positive integers. Let $n=n_{1}+\cdots+n_{t}$ and $\theta_{1} \geq \cdots \geq \theta_{n}$ be the Seidel eigenvalues of $K_{n_{1}, \ldots, n_{t}}$. Then

$$
\mathcal{E}\left(S\left(K_{n_{1}, \ldots, n_{t}}\right)\right)=2\left(n_{1}+\cdots+n_{t}\right)-2 t-2 \theta_{n} .
$$

In other words,

$$
\left|\theta_{1}\right|+\cdots+\left|\theta_{n}\right|=2 n-2 t-2 \theta_{n}
$$

Proof. For $t=2$, the result follows by Remark 1. Thus let $t \geq 3$. Without losing the generality assume that $n_{1} \geq n_{2} \geq \cdots \geq n_{t}$. Let $G=K_{n_{1}, \ldots, n_{t}}$. We note that the blocks of the Seidel matrix of $G$, the blocks of $S(G)$, are 
$J-I$ and $-J$. In fact one can see that

$$
S(G)+I=\left[\begin{array}{ccccc}
J_{1,1} & -J_{1,2} & \cdots & -J_{1, t-1} & -J_{1, t} \\
-J_{2,1} & J_{2,2} & \cdots & -J_{2, t-1} & -J_{2, t} \\
\vdots & & \ddots & & \vdots \\
-J_{t-1,1} & -J_{t-1,2} & \cdots & J_{t-1, t-1} & -J_{t-1, t} \\
-J_{t, 1} & -J_{t, 2} & \cdots & -J_{t, t-1} & J_{t, t}
\end{array}\right]_{t \times t}
$$

where for $1 \leq i, j \leq t, J_{i, j}$ is the $n_{i} \times n_{j}$ matrix that all of entries are 1. It is not hard to see that $\operatorname{rank}(S(G)+I)=t$. Since $S(G)$ is a symmetric $n \times n$ matrix and $\operatorname{rank}(S(G)+I)=t$, we obtain that the multiplicity of zero as an eigenvalue of $S(G)+I$ is $n-t$. Hence the multiplicity of -1 as an eigenvalue of $S(G)$ is $n-t$. Let

$$
D(G)=\left[\begin{array}{ccccc}
J_{1,1} & O_{1,2} & \cdots & O_{1, t-1} & O_{1, t} \\
O_{2,1} & J_{2,2} & \cdots & O_{2, t-1} & O_{2, t} \\
\vdots & & \ddots & & \vdots \\
O_{t-1,1} & O_{t-1,2} & \cdots & J_{t-1, t-1} & O_{t-1, t} \\
O_{t, 1} & O_{t, 2} & \cdots & O_{t, t-1} & J_{t, t}
\end{array}\right]_{t \times t},
$$

where for $1 \leq i, j \leq t, O_{i, j}$ is the $n_{i} \times n_{j}$ matrix that all of entries are zero. Therefore $S(G)+I=2 D(G)-J$. In other words,

$$
-S(G)-I=-2 D(G)+J .
$$

Since $\theta_{1} \geq \cdots \geq \theta_{n}$ are the eigenvalues of $S(G)$, we find that the eigenvalues of $-S(G)-I$ are

$$
-1-\theta_{n} \geq-1-\theta_{n-1} \geq \cdots \geq-1-\theta_{1} .
$$

We note that for $i=1, \ldots, t$, the eigenvalues of $J_{i, i}$ are $n_{i}$ (with multiplicity 1 ) and 0 with multiplicity $n_{i}-1$. Hence the eigenvalues of $-2 D(G)$ are

$$
0 \geq 0 \geq \cdots \geq 0 \geq-2 n_{t} \geq-2 n_{t-1} \geq \cdots \geq-2 n_{1},
$$

where the multiplicity of zero as an eigenvalue of $-2 D(G)$ is $n-t$. By Lemma 1 and Equation (3), we find that for $i=1, \ldots, n, \lambda_{i}(-S(G)-I)=\lambda_{i}(-2 D(G)+J) \geq \lambda_{i}(-2 D(G))$ and for $i=1, \ldots, n-1, \lambda_{i}(-2 D(G)) \geq$ $\lambda_{i+1}(-2 D(G)+J)=\lambda_{i+1}(-S(G)-I)$. Thus by Equations (4) and (5) we conclude that

$$
\begin{aligned}
& -1-\theta_{n} \geq 0 \geq-1-\theta_{n-1} \geq 0 \geq \cdots \geq-1-\theta_{t+1} \geq 0 \\
& \geq-1-\theta_{t} \geq-2 n_{t} \geq-1-\theta_{t-1} \geq-2 n_{t-1} \geq \cdots \geq-1-\theta_{1} \geq-2 n_{1} .
\end{aligned}
$$

This implies that (by multiplying by -1 and adding by -1 )

$$
\begin{aligned}
& 2 n_{1}-1 \geq \theta_{1} \geq 2 n_{2}-1 \geq \theta_{2} \geq \cdots \geq 2 n_{t-1}-1 \geq \theta_{t-1} \geq 2 n_{t}-1 \geq \theta_{t} \geq-1 \\
& \geq \theta_{t+1} \geq-1 \geq \theta_{t+2} \geq-1 \geq \cdots \geq-1 \geq \theta_{n-1} \geq-1 \geq \theta_{n} .
\end{aligned}
$$

This shows that

$$
\theta_{1} \geq \theta_{2} \geq \cdots \geq \theta_{t-1} \geq 2 n_{t}-1 \geq 1
$$

and

$$
\theta_{t+1}=\theta_{t+2}=\cdots=\theta_{n-1}=-1 .
$$

Since by Theorem 4, $\theta_{n}<-1$ and the multiplicity of -1 as an eigenvalue of $S(G)$ is exactly $n-t$, by Equations (8) and (9) we conclude that $\theta_{t}=-1$. Now by Equations (8) and (9) we can compute the Seidel energy of complete multipartite graph $K_{n_{1}, \ldots, n_{t}}$. In fact

$$
\mathcal{E}\left(S\left(K_{n_{1}, \ldots, n_{t}}\right)\right)=\left|\theta_{1}\right|+\cdots+\left|\theta_{t-1}\right|+\left|\theta_{t}\right|+\cdots+\left|\theta_{n-1}\right|+\left|\theta_{n}\right|
$$




$$
=\theta_{1}+\cdots+\theta_{t-1}+n-t-\theta_{n} .
$$

On the other hand, since $\operatorname{tr}(S(G))=0$ and $\theta_{t}=\theta_{t+1}=\cdots=\theta_{n-1}=-1$, we find that $\theta_{1}+\cdots+\theta_{t-1}-(n-t)+\theta_{n}=0$. Hence

$$
\theta_{1}+\cdots+\theta_{t-1}=n-t-\theta_{n}
$$

Now by combining Equations (10) and (11) we find that

$$
\mathcal{E}\left(S\left(K_{n_{1}, \ldots, n_{t}}\right)\right)=2 n-2 t-2 \theta_{n} .
$$

In [5], Haemers conjectured that among all simple graphs with $n$ vertices, the complete graph $K_{n}$ has the minimum Seidel energy. Now by applying Theorem 5, we prove that this conjecture is true among the family of complete multipartite graphs. We note that by Remark 1, the Seidel energy of the complete graph $K_{n}$ is equal to $2 n-2$.

Theorem 6. Let $t \geq 2$ and $n_{1}, \ldots, n_{t}$ be some positive integers and $n=n_{1}+\cdots+n_{t}$. Then

$$
\mathcal{E}\left(S\left(K_{n_{1}, \ldots, n_{t}}\right)\right) \geq \mathcal{E}\left(S\left(K_{n}\right)\right) \text {. }
$$

Moreover the equality holds if and only if $t=2$ or $t \geq 3$ and $n_{1}=\cdots=n_{t}=1$.

Proof. If $t=2$, then by Remark $1, \mathcal{E}\left(S\left(K_{n_{1}, n_{2}}\right)\right)=2\left(n_{1}+n_{2}\right)-2=2 n-2=\mathcal{E}\left(S\left(K_{n}\right)\right)$. Now let $t \geq 3$. If $n_{1}=\cdots=n_{t}=1$, then $n=t$ and $K_{n_{1}, \ldots, n_{t}}=K_{t}$. Hence by Remark $1, \mathcal{E}\left(S\left(K_{n_{1}, \ldots, n_{t}}\right)\right)=\mathcal{E}\left(S\left(K_{t}\right)\right)=2 t-2$. So to compete the proof it suffices to show that if $t \geq 3$ and $\max \left\{n_{1}, \ldots, n_{t}\right\} \geq 2$, then $\mathcal{E}\left(S\left(K_{n_{1}, \ldots, n_{t}}\right)\right)>\mathcal{E}\left(S\left(K_{n}\right)\right)$. Suppose that $t \geq 3$ and $\max \left\{n_{1}, \ldots, n_{t}\right\} \geq 2$. Let $\theta_{n}=\theta_{n}\left(K_{n_{1}}, \ldots, n_{t}\right)$. Using Theorems 4 and 5 we obtain that

$$
\mathcal{E}\left(S\left(K_{n_{1}, \ldots, n_{t}}\right)\right)=2 n-2 t-2 \theta_{n}>2 n-2 t+2(t-1)=2 n-2=\mathcal{E}\left(S\left(K_{n}\right)\right) .
$$

Thus $\mathcal{E}\left(S\left(K_{n_{1}, \ldots, n_{t}}\right)\right)>\mathcal{E}\left(S\left(K_{n}\right)\right)$. The proof is complete.

Data Availability Statement: Data sharing is not applicable to this article as no datasets were generated or analysed during the current study.

\section{References}

[1] A. Berman, N. Shaked-Monderer, R. Singh, X.D.Zhang, Complete multipartite graphs that are determined, up to switching, by their Seidel spectrum, Linear Algebra Appl. 564 (2019) 58-71.

[2] A.E. Brouwer, W.H. Haemers, Spectra of graphs, Springer, New York, 2012.

[3] I. Gutman, The energy of a graph, Ber. Math. Statist. Sekt. Forsch. Graz 103 (1978) 1-22.

[4] I. Gutman, The energy of a graph: old and new results, in: A. Betten, A. Kohnert, R. Laue, A. Wassermann (Eds.), Algebraic Combinatorics and Applications, Springer, Berlin, 2001, pp. 196-211.

[5] W.H. Haemers, Seidel switching and graph energy, MATCH Commun. Math. Comput. Chem. 68 (2012) 653-659.

[6] M.R. Oboudi, A relation between the signless Laplacian spectral radius of complete multipartite graphs and majorization, Linear Algebra Appl. 565 (2019) 225-238.

[7] M.R. Oboudi, Cospectrality of complete bipartite graphs, Linear Multilinear Algebra 64 (2016) 2491-2497.

[8] M.R. Oboudi, Energy and Seidel energy of graphs, MATCH Commun. Math. Comput. Chem. 75 (2016) 291-303.

[9] H.S. Ramane, I. Gutman, M.M. Gundloor, Seidel energy of iterated line graphs of regular graphs, Kragujevac J. Math. 39 (2015) 7-12.

[10] D. Stevanović, I. Gutman, M.U. Rehman, On spectral radius and energy of complete multipartite graphs, Ars Math. Contemp. 9 (2015) 109-113.

[11] G. Yu, Y.Wu, J. Shu, Signless Laplacian spectral radii of graphs with given chromatic number, Linear Algebra Appl. 435 (2011) $1813-1822$.

[12] L. Wang, X. Liu, Integral complete multipartite graphs, Discrete Math. 308 (2008) 3860-3870.

[13] L. Wang, G. Zhao, K. Li, Seidel Integral Complete r-Partite Graphs, Graphs Combin. 30 (2014) 479-493. 